\title{
A Finite Element Variational Multiscale Method Based on Two Local Gauss Integrations for Stationary Conduction-Convection Problems
}

\author{
Yu Jiang, ${ }^{1}$ Liquan Mei, ${ }^{1,2}$ Huiming Wei, ${ }^{3}$ Weijun Tian, ${ }^{1,4}$ \\ and Jiatai $\mathbf{G e}^{1}$ \\ ${ }^{1}$ School of Mathematics and Statistics, Xi'an Jiaotong University, Xi'an 710049, China \\ ${ }^{2}$ Center for Computational Geosciences, Xi'an Jiaotong University, Xi'an 710049, China \\ ${ }^{3}$ China Nuclear Power Simulation Technology Company Limited, Shenzhen 518115, China \\ ${ }^{4}$ College of Mathematics and Information Science, Xianyang Normal University, Xianyang 712000, China
}

Correspondence should be addressed to Liquan Mei, lqmei@mail.xjtu.edu.cn

Received 6 July 2012; Revised 23 October 2012; Accepted 24 October 2012

Academic Editor: Hung Nguyen-Xuan

Copyright (C) 2012 Yu Jiang et al. This is an open access article distributed under the Creative Commons Attribution License, which permits unrestricted use, distribution, and reproduction in any medium, provided the original work is properly cited.

A new finite element variational multiscale (VMS) method based on two local Gauss integrations is proposed and analyzed for the stationary conduction-convection problems. The valuable feature of our method is that the action of stabilization operators can be performed locally at the element level with minimal additional cost. The theory analysis shows that our method is stable and has a good precision. Finally, the numerical test agrees completely with the theoretical expectations and the " exact solution," which show that our method is highly efficient for the stationary conductionconvection problems.

\section{Introduction}

The conduction-convection problems constitute an important system of equations in atmospheric dynamics and dissipative nonlinear system of equations. Many authors have worked on these problems [1-8]. The governing equations couple viscous incompressible flow and heat transfer process [9], where the incompressible fluid is the Boussinesq approximation to the nonstationary Navier-Stokes equations. Christon et al. [10] summarized some relevant results for the fluid dynamics of thermally driven cavity. A multigrid (MG) technique was applied for the conduction-convection problems [11, 12]. Luo et al. [13] combined proper orthogonal decomposition (POD) with the Petrov-Galerkin least squares mixed finite element (PLSMFE) method for the problems. In [14], a Newton iterative mixed finite element method 
for the stationary conduction-convection problems was shown by Si et al. In [15], Si and He gave a defect-correction mixed finite element method for the stationary conduction-convection problems. In [3], an analysis of conduction natural convection conjugate heat transfer in the gap between concentric cylinders under solar irradiation was carried out. In [16], Boland and Layton gave an error analysis for finite element methods for steady natural convection problems. Variational multiscale (VMS) method which defines the large scales in a different way, namely, by a projection into appropriate subspaces, see Guermond [17], Hughes et al. [18-20] and Layton [21], and other literatures on VMS methods [22-24]. The new finite element VMS strategy requires edge-based data structure and a subdivision of grids into patches. It does not require a specification of mesh-dependent parameters and edge-based data structure, and it is completely local at the element level. Consequently, the new VMS method under consideration can be integrated in existing codes with very little additional coding effort.

For the conduction-convection problems, we establish such system that $\Omega$ be a bounded domain in $R^{d}(d=2$ or 3$)$, with Lipschitz-continuous boundary $\partial \Omega$. In this paper, we consider the stationary conduction-convection problem as follows:

$$
\begin{gathered}
-2 v \nabla \cdot D(u)+(u \cdot \nabla u)+\nabla p=\lambda j T, \quad x \in \Omega, \\
\nabla \cdot u=0, \quad x \in \Omega, \\
-\Delta T+\lambda u \cdot \nabla T=0, \quad x \in \Omega, \\
u=0, \quad T=T_{0}, \quad x \in \partial \Omega,
\end{gathered}
$$

where $D(u)=\left(\nabla u+\nabla u^{T}\right) / 2$ is the velocity deformation tensor, $(u, p, T) \in X \times M \times W, \Omega \subset R^{d}$ is a bounded convex domain. $u=\left(u_{1}(x), u_{2}(x)\right)^{T}$ represents the velocity vector, $p(x)$ the pressure, $T(x)$ the temperature, $\lambda>0$ the Grashoff number, $j=(0,1)^{T}$ the two-dimensional vector and $v>0$ the viscosity.

The study is organized as follows. In the next section, the finite element VMS method is given. In Section 3, we give the stability. The error analysis is given in Section 4. In Section 5, we show some numerical test. The last but not least is the conclusion given in Section 6 .

\section{Finite Element VMS Method}

Here, we introduce some notations

$$
X=H_{0}^{1}(\Omega)^{d}, \quad M=L_{0}^{2}(\Omega)=\left\{\varphi \in L^{2}(\Omega) ; \int_{\Omega} \varphi d x=0\right\}, \quad W=H^{1}(\Omega) .
$$

For $h>0$, finite-dimension subspace $\left(X_{h}, M_{h}, W_{h}\right) \subset(X, M, W)$ is introduced which is associated with $\Omega_{e}$, a triangulation of $\Omega$ into triangles or quadrilaterals, assumed to be regular in the usual sense. In this study, the finite-element subspaces of personal preference are defined by setting the continuous piecewise (bi)linear velocity and pressure subspace, let $\tau_{h}$ be the regular triangulations or quadrilaterals of the domain $\Omega$ and define the mesh parameter $h=$ $\max _{\Omega_{e} \in \tau_{h}}\left\{\operatorname{diam}\left(\Omega_{e}\right)\right\}$,

$$
\begin{aligned}
& X_{h}=\left\{v \in X:\left.v\right|_{\Omega_{e}} \in R_{l}\left(\Omega_{e}\right)^{d} \forall \Omega_{e} \in \tau_{h}\right\}, \\
& M_{h}=\left\{q \in M:\left.q\right|_{\Omega_{e}} \in R_{l}\left(\Omega_{e}\right) \forall \Omega_{e} \in \tau_{h}\right\}, \\
& W_{h}=\left\{\phi \in M:\left.\phi\right|_{\Omega_{e}} \in R_{l}\left(\Omega_{e}\right) \forall \Omega_{e} \in \tau_{h}\right\},
\end{aligned}
$$


where $W_{0 h}=W_{h} \cap H_{0}^{1}, l \geq 1$ is integers. $R_{l}\left(\Omega_{e}\right)=P_{l}\left(\Omega_{e}\right)$ if $\Omega_{e}$ is triangular and $R_{l}\left(\Omega_{e}\right)=$ $Q_{l}\left(\Omega_{e}\right)$ if $\Omega_{e}$ is quadrilateral. Here $\left(X_{h}, M_{h}\right)$ does not satisfy the discrete LadyzhenskayaBabuška-Brezzi (LBB) condition

$$
\sup _{v_{h} \in X_{h}} \frac{d\left(v_{h}, p_{h}\right)}{\left\|\nabla v_{h}\right\|_{0}} \geq \beta\left\|p_{h}\right\|_{0^{\prime}} \quad \forall p_{h} \in M_{h} .
$$

Now, in order to stabilize the convective term appropriately for the higher Reynolds number and avoid the extra storage, we supply finite element VMS method that the local stabilization form of the difference between a consistent and an underintegrated mass matrices based on two local Gauss integrations at element level as the stabilize term

$$
G\left(p_{h}, q_{h}\right)=\epsilon_{d}\left(a_{k}\left(p_{h}, q_{h}\right)-a_{1}\left(p_{h}, q_{h}\right)\right)
$$

Here,

$$
\begin{gathered}
a_{k}\left(p_{h}, q_{h}\right)=p_{G}^{T} M_{k} q_{G}, \quad a_{1}\left(p_{h}, q_{h}\right)=p_{G}^{T} M_{1} q_{G}, \\
p_{G}^{T}=\left[p_{1}, p_{2} \ldots, p_{N}\right]^{T}, \quad q_{G}=\left[q_{1}, q_{2}, \ldots, q_{N}\right], \\
M_{i j}=\left(\phi_{i}, \phi_{j}\right), \quad p_{h}=\sum_{i=1}^{N} p_{i} \phi_{i}, \quad p_{i}=p_{h}\left(x_{i}\right), \forall p_{h} \in M_{h}, i=1,2, \ldots, N, \\
M_{k}=\left(M_{i j}^{k}\right)_{N \times N^{\prime}} \quad M_{1}=\left(M_{i j}^{1}\right)_{N \times N^{\prime}}
\end{gathered}
$$

the stabilization parameter $\epsilon_{d}\left(\epsilon_{d}=o(h)\right)$ in this scheme acts only on the small scales, $\phi_{i}$ is the basis function of the velocity on the domain $\Omega$ such that its value is one at node $x_{i}$ and zero at other nodes, and $N$ is the dimension of $M_{h}$. The symmetric and positive matrices $M_{i j}^{k}, k \geq 2$ and $M_{i j}^{1}$ are the stiffness matrices computed by using $k$-order and 1-order Gauss integrations at element level, respectively. $p_{i}$ and $q_{i}, i=1,2, \ldots, N$ are the values of $p_{h}$ and $q_{h}$ at the node $x_{i}$. In detail, the stabilized term can be rewritten as

$$
\begin{gathered}
G\left(p_{h}, q_{h}\right)=\epsilon_{d} \sum_{\Omega_{e} \in \tau_{h}}\left\{\int_{\Omega_{e}, k} p_{h} q_{h} d x-\int_{\Omega_{e}, 1} p_{h} q_{h} d x\right\}, \quad \forall p_{h}, q_{h} \in M_{h}, \\
G(p, q)=\left(p-\coprod_{h} p, q-\coprod_{h} q\right) .
\end{gathered}
$$

$L^{2}$-projection operator $\amalg_{h}: L^{2}(\Omega) \rightarrow R_{0}$ with the following properties [25]:

$$
\begin{gathered}
\left(p, q_{h}\right)=\left(\coprod_{h} p, q_{h}\right), \quad \forall p \in M, q_{h} \in R_{0} ; \\
\left\|\coprod_{h} p\right\|_{0} \leq c\|p\|_{0}, \quad \forall p \in M ; \\
\left\|p-\coprod_{h} p\right\|_{0} \leq c h\|p\|_{1}, \quad \forall p \in H^{1}(\Omega) \cap M .
\end{gathered}
$$


Lemma 2.1 (see [26]). Let $\left(X_{h}, M_{h}\right)$ be defined as above, then there exists a positive constant $\beta$ independent of $h$, such that

$$
\begin{gathered}
|B((u, p) ;(v, q))| \leq c\left(\|u\|_{1}+\|p\|_{0}\right)\left(\|v\|_{1}+\|q\|_{0}\right)(u, p), \quad(v, q) \in(X, M), \\
\beta\left(\left\|u_{h}\right\|_{1}+\left\|v p_{h}\right\|_{0}\right) \leq \sup _{\left(v_{h}, q_{h}\right) \in\left(X_{h}, M_{h}\right)} \frac{\left|B\left(\left(u_{h}, p_{h}\right) ;\left(v_{h}, q_{h}\right)\right)\right|}{\|v\|_{1}+\|q\|_{0}}, \quad \forall\left(u_{h}, p_{h}\right) \in\left(X_{h}, M_{h}\right), \\
|G(p, q)| \leq C\left\|\left(I-I I_{h}\right) p\right\|_{0}\left\|\left(I-I I_{h}\right) q\right\|_{0}, \quad \forall p, q \in M .
\end{gathered}
$$
follows.

Using the above notations, the VMS variational formulation of problems (1.1) reads as Find $\left(\mathrm{A}_{1}\right)\left(u_{h}, p_{h}, T_{h}\right) \in X_{h} \times M_{h} \times W_{h}$ such that

$$
\begin{gathered}
a\left(u_{h}, v_{h}\right)-d\left(p_{h}, v_{h}\right)+d\left(q_{h}, u_{h}\right)+b\left(u_{h}, u_{h}, v_{h}\right)+G\left(p_{h}, q_{h}\right)=\lambda\left(j T_{h}, v_{h}\right), \quad \forall v_{h} \in X_{h}, \varphi_{h} \in M_{h} \\
\bar{a}\left(T_{h}, \psi_{h}\right)+\lambda \bar{b}\left(u_{h}, T_{h}, \psi_{h}\right)=0, \quad \forall \psi_{h} \in W_{0 h} .
\end{gathered}
$$

Given $\left(\mathrm{A}_{2}\right)\left(u_{h}^{n-1}, T_{h}^{n-1}\right)$, find $\left(u_{h^{\prime}}^{n} p_{h^{\prime}}^{n} T_{h}^{n}\right) \in X_{h} \times M_{h} \times W_{h}$ such that

$$
\begin{gathered}
a\left(u_{h^{\prime}}^{n} v_{h}\right)-d\left(p_{h^{\prime}}^{n} v_{h}\right)+d\left(q_{h}, u_{h}^{n}\right)+b\left(u_{h}^{n}, u_{h}^{n-1}, v_{h}\right)+b\left(u_{h}^{n-1}, u_{h}^{n}, v_{h}\right)+G\left(p_{h^{\prime}}^{n} q_{h}\right) \\
=b\left(u_{h}^{n-1}, u_{h}^{n-1}, v_{h}\right)+\lambda\left(j T_{h}^{n}, v_{h}\right), \quad \forall v_{h} \in X_{h}, \varphi_{h} \in M_{h} \\
\bar{a}\left(T_{h}^{n}, \psi_{h}\right)+\lambda \bar{b}\left(u_{h}^{n-1}, T_{h}^{n}, \psi_{h}\right)=0, \quad \forall \psi_{h} \in W_{0 h}
\end{gathered}
$$

where $a(u, v)=v(\nabla u, \nabla v), \bar{a}(T, \psi)=(\nabla T, \nabla \psi) d(q, v)=(q, \operatorname{div} v)$, and

$$
\begin{aligned}
& b(u, v, w)=((u \cdot \nabla v), w)+\frac{1}{2}((\operatorname{div} u) v, w)=\frac{1}{2}((u \cdot \nabla) v, w)-\frac{1}{2}((u \cdot \nabla) w, v), \\
& \bar{b}(u, T, \psi)=((u \cdot \nabla T), w)+\frac{1}{2}((\operatorname{div} u) T, \psi)=\frac{1}{2}((u \cdot \nabla) T, \psi)-\frac{1}{2}((u \cdot \nabla) \psi, T) .
\end{aligned}
$$

$\left(B_{1}\right)$ There exists a constant $C$ which only depends on $\Omega$, such that

(i) $\|u\|_{0} \leq C\|\nabla u\|_{0},\|u\|_{0,4} \leq C\|\nabla u\|_{0}$, for all $u \in H_{0}^{1}(\Omega)^{d}$ (or $H_{0}^{1}(\Omega)$ ),

(ii) $\|u\|_{0,4} \leq C\|u\|_{1}$, for all $u \in H^{1}(\Omega)^{d}$

(iii) $\|u\|_{0,4} \leq 2^{1 / 2}\|\nabla u\|_{0}^{1 / 2}\|u\|_{0}^{1 / 2}$, for all $u \in H_{0}^{1}(\Omega)^{d}$ (or $H_{0}^{1}(\Omega)$ )

$\left(\mathrm{B}_{2}\right)$ Assuming $\partial \Omega \in C^{k, \alpha}(k \geq 0, \alpha>0)$, then, for $T_{0} \in C^{k, \alpha}(\partial \Omega)$, there exists an extension $T_{0}$ in $C_{0}^{k, \alpha}\left(R^{d}\right)$, such that

$$
\left\|T_{0}\right\|_{k, q} \leq \varepsilon, \quad k \geq 0,1 \leq q \leq \infty,
$$

where $\varepsilon$ is an arbitrary positive constant. 
$\left(\mathrm{B}_{3}\right) b(\cdot, \cdot, \cdot)$ and $\bar{b}(\cdot, \cdot, \cdot)$ have the following properties.

(i) For all $u \in X, v, w \in X, T \cdot \varphi \in H_{0}^{1}(\Omega)$, there holds that

$$
b(u, v, w)=-b(u, w, v), \quad \bar{b}(u, T, \psi)=-\bar{b}(u, \psi, T) .
$$

(ii) For all $u \in X, v \in H^{1}(\Omega)^{d}, T \in H^{1}(\Omega)$, for all $w \in X$ (or $\varphi \in H_{0}^{1}(\Omega)$ ), there holds that

$$
\begin{aligned}
& |b(u, v, w)| \leq N\|\nabla u\|_{0}\|\nabla v\|_{0}\|\nabla w\|_{0}, \\
& |\bar{b}(u, T, \varphi)| \leq \bar{N}\|\nabla u\|_{0}\|\nabla T\|_{0}\|\nabla \varphi\|_{0^{\prime}}
\end{aligned}
$$

where $N=\sup _{u, v, w}|b(u, v, w)| /\|\nabla u\|_{0}\|\nabla v\|_{0}\|\nabla w\|_{0}, \bar{N}=\sup _{u, v, w}|\bar{b}(u, T, \varphi)| /\|\nabla u\|_{0}\|\nabla T\|_{0}$ $\|\nabla \varphi\|_{0}$.

\section{Stability Analysis}

Lemma 3.1. The trilinear form $b$ satisfies the following estimate:

$$
\left|b\left(u_{h}, v_{h}, w\right)\right|+\left|b\left(v_{h}, u_{h}, w\right)\right|+\left|b\left(w, u_{h}, v_{h}\right)\right| \leq C|\log h|^{1 / 2}\left\|\nabla u_{h}\right\|_{0}\left\|\nabla v_{h}\right\|_{0}\|w\|_{0} .
$$

Theorem 3.2. Suppose that $\left(\mathrm{B}_{1}\right)-(\mathrm{B} 3)$ are valid and $\varepsilon$ is a positive constant number, such that

$$
\frac{64 C^{2} N \lambda \varepsilon}{3 v^{2}}<1, \quad \frac{16 C^{2} \lambda^{2} \bar{N} \varepsilon}{3 v}<1, \quad\left\|\nabla T_{0}\right\|_{0} \leq \frac{\varepsilon}{4}, \quad\left\|T_{0}\right\|_{0} \leq \frac{C \varepsilon}{4} .
$$

Then $\left(u_{h}^{m}, T_{h}^{m}\right)$ defined by $\left(\mathrm{A}_{2}\right)$ satisfies

$$
\left\|\nabla u_{h}^{m}\right\|_{0} \leq \frac{8 C^{2} \lambda \varepsilon}{3 v}, \quad\left\|\nabla T_{h}^{m}\right\|_{0} \leq \varepsilon
$$

Proof. We prove this theorem by the inductive method. For $m=1$, (3.3) holds obviously. Assuming that (3.3) holds for $m=n-1$, we want to prove that it holds for $m=n$. We estimate $\left\|\Delta u_{h}^{n}\right\|$ firstly. Letting $v_{h}=u_{h}^{n}, q_{h}=0$ in the first equation of (2.10) and using (2.13), we get

$$
a\left(u_{h}^{n}, u_{h}^{n}\right)+b\left(u_{h}^{n}, u_{h}^{n-1}, u_{h}^{n}\right)=b\left(u_{h}^{n-1}, u_{h}^{n-1}, u_{h}^{n}\right)+\lambda\left(j T_{h}^{n}, u_{h}^{n}\right)
$$

Setting $T_{h}^{n-1}=k_{h}^{n-1}+T_{0}$ and using (2.14), we have

$$
v\left\|\nabla u_{h}^{n}\right\|_{0} \leq N\left\|\nabla u_{h}^{n}\right\|_{0}\left\|\nabla u_{h}^{n-1}\right\|_{0}+N\left\|\nabla u_{h}^{n-1}\right\|_{0}^{2}+C^{2} \lambda\left\|\nabla k_{h}^{n-1}\right\|_{0}+C \lambda\left\|\nabla T_{0}\right\|_{0} .
$$


Letting $T_{h}^{n}=k_{h}^{n}+T_{0}, \psi=k_{h}^{n}$ in the second equation of (2.10), we can obtain

$$
\bar{a}\left(k_{h}^{n}, k_{h}^{n}\right)=-\lambda \bar{b}\left(u_{h}^{n}, T_{0}, k_{h}^{n}\right)-\bar{a}\left(T_{0}, k_{h}^{n}\right)
$$

Using (2.12), (2.14), and (3.2), we get

$$
\begin{gathered}
\left\|\nabla k_{h}^{n-1}\right\|_{0} \leq \lambda \bar{N}\left\|\nabla u_{h}^{n-1}\right\|_{0}\left\|\nabla T_{0}\right\|_{0}+\left\|\nabla T_{0}\right\|_{0} \\
\leq \frac{\lambda \bar{N} \varepsilon}{4}\left\|\nabla u_{h}^{n-1}\right\|_{0}+\left\|\nabla T_{0}\right\|_{0} \leq \frac{3 \varepsilon}{8} \leq \frac{3 \varepsilon}{4} \\
\left(v-N\left\|\nabla u_{h}^{n-1}\right\|_{0}\right)\left\|\nabla u_{h}^{n}\right\|_{0} \leq N\left\|\nabla u_{h}^{n-1}\right\|_{0}^{2}+C^{2} \lambda \varepsilon \\
\leq C^{2} \lambda \varepsilon+\frac{64 C^{4} N}{9 v^{2}} \lambda^{2} \varepsilon^{2} \leq \frac{4 C^{2} \lambda \varepsilon}{3} .
\end{gathered}
$$

Using (3.2), we have $v-N\left\|\nabla u_{h}^{n-1}\right\|_{0} \geq 7 v / 8$. Then,

$$
\left\|\nabla u_{h}^{n}\right\|_{0} \leq \frac{8 C^{2} \lambda \varepsilon}{3 v}
$$

Combining (2.12), (2.14), (3.2), and (3.6), we arrive at

$$
\begin{gathered}
\left\|\nabla k_{h}^{n}\right\|_{0} \leq \lambda \bar{N}\left\|\nabla u_{h}^{n}\right\|_{0}\left\|\nabla T_{0}\right\|_{0}+\left\|\nabla T_{0}\right\|_{0} \leq \frac{3 \varepsilon}{4}, \\
\left\|\nabla T_{h}^{n}\right\|_{0} \leq\left\|\nabla k_{h}^{n}\right\|_{0}+\left\|\nabla T_{0}\right\|_{0} \leq \varepsilon .
\end{gathered}
$$

Therefore, we finish the proof.

\section{Error Analysis}

In this section, we establish the $H^{1}$-bound of the error $u_{h}^{n}-u, T_{h}^{n}-T$ and $L^{2}$-bounds of the error $p_{h}^{n}-p$. Setting $\left(e^{n}, \mu^{n}, \eta^{n}\right)=\left(u_{h}^{n}-u_{h}, p_{h}^{n}-p_{h}, T_{h}^{n}-T_{h}\right)$. Firstly, we give some Lemmas.

Lemma 4.1. In [4], If $\mathrm{B}_{1}-\mathrm{B}_{3}$ hold, $(u, p, T) \in H^{m+1}(\Omega) \times H^{m}(\Omega) \times H^{m+1}(\Omega)$ and $\left(u_{h}, p_{h}, T_{h}\right) \in X_{h} \times$ $M_{h} \times W_{h}$ are the solution of problem $\left(\mathrm{A}_{1}\right)$ and $\left(\mathrm{A}_{2}\right)$, respectively, then there holds that

$$
\left\|\nabla\left(u-u_{h}\right)\right\|_{0}+\left\|p-p_{h}\right\|_{0}+\left\|\nabla\left(T-T_{h}\right)\right\|_{0} \leq C h^{m}\left(\|u\|_{m+1}+\|p\|_{m}+\|T\|_{m+1}\right) .
$$

Lemma 4.2. Under the assumptions of Theorem 3.2, $\left(\mathrm{A}_{2}\right)$ has a unique solution $\left(u_{h}, p_{h}, T_{h}\right) \in X_{h} \times$ $M_{h} \times W_{h}$, such that $\left.T\right|_{\partial \Omega}=T_{0}$ and

$$
\left\|\nabla u_{h}\right\|_{0} \leq \frac{8 C^{2} \lambda \varepsilon}{3 v}, \quad\left\|\nabla T_{h}\right\|_{0} \leq \varepsilon
$$

The detail proof we can see $[4,13,14]$. 
Theorem 4.3. Under the assumption of Theorem 3.2, there holds

$$
\begin{gathered}
\left\|\nabla e^{n}\right\|_{0} \leq \frac{C^{2} \lambda \varepsilon}{2^{n-3} 3 v}, \quad\left\|\nabla \eta^{n}\right\|_{0} \leq \frac{\varepsilon}{2^{n+1}}, \\
\left\|\mu^{n}\right\|_{0} \leq \beta^{-1} \begin{cases}\frac{v \varepsilon}{2}+\frac{4 C^{2} \lambda \varepsilon}{3}, & n=1 \\
(v+2 N \varepsilon) \frac{C^{2} \lambda \varepsilon}{2^{n-3} 3 v}+N\left(\frac{C^{2} \lambda \varepsilon}{2^{n-4} 3 v}\right)^{2}+\frac{C^{2} \lambda \varepsilon}{2^{n}}, & n \geq 2 .\end{cases}
\end{gathered}
$$

Proof. Subtracting (2.10) from (2.9), we get the following error equations, namely $\left(e^{n}, \mu^{n}, \eta^{n}\right)$ satisfies

$$
\begin{gathered}
a\left(e^{n}, v_{h}\right)-d\left(\mu^{n}, v_{h}\right)+d\left(q_{h}, e^{n}\right)+b\left(e^{n}, u_{h}^{n-1}, v_{h}\right)+b\left(u_{h}^{n-1}, e^{n}, v_{h}\right)+G\left(\mu^{n}, q_{h}\right) \\
=b\left(e^{n-1}, e^{n-1}, v_{h}\right)+\lambda\left(j \eta^{n}, v_{h}\right) \\
\bar{a}\left(\eta^{n}, \psi_{h}\right)+\lambda \bar{b}\left(e^{n}, T_{h}^{n}, \psi_{h}\right)+\lambda \bar{b}\left(u_{h}^{n-1}, \eta^{n}, q_{h}\right)=0
\end{gathered}
$$

Here, let $\psi_{h}=\eta^{n}$, in (4.5), then we have

$$
\bar{a}\left(\eta^{n}, \eta^{n}\right)+\lambda \bar{b}\left(e^{n}, T_{h}^{n}, \eta^{n}\right)=0
$$

By using (2.14), we get

$$
\left\|\nabla \eta^{n}\right\|_{0} \leq \lambda \bar{N} \varepsilon\left\|\nabla e^{n}\right\|_{0}
$$

In (4.4), we take $v_{h}=e^{n} \in X_{h}, q_{h}=\mu^{n}$, then

$$
a\left(e^{n}, e^{n}\right)+b\left(e^{n}, u_{h}^{n-1}, e^{n}\right)+b\left(u_{h}^{n-1}, e^{n}, e^{n}\right)+G\left(\mu^{n}, \mu^{n}\right)=b\left(e^{n-1}, e^{n-1}, e^{n}\right)+\lambda\left(j \eta^{n}, e^{n}\right)
$$

Using (2.13) and (2.14), we have

$$
v\left\|\nabla e^{n}\right\|_{0}+G\left(\mu^{n}, \mu^{n}\right) \leq N\left\|\nabla e^{n}\right\|_{0}\left\|\nabla u_{h}^{n-1}\right\|_{0}+N\left\|\nabla e^{n-1}\right\|_{0}^{2}+C^{2} \lambda\left\|\nabla \eta^{n}\right\|_{0^{\prime}}
$$

then, we obtain

$$
\left(v-N\left\|\nabla u_{h}^{n-1}\right\|_{0}\right)\left\|\nabla e^{n}\right\|_{0} \leq N\left\|\nabla e^{n-1}\right\|_{0}^{2}+C^{2} \lambda\left\|\nabla \eta^{n}\right\|_{0}
$$


By using $v-N\left\|\nabla u_{h}^{n-1}\right\|_{0} \geq 7 v / 8$. Equations (3.3) and (4.2), we get

$$
\begin{aligned}
& \frac{7}{8} \mathcal{v}\left\|\nabla e^{n}\right\|_{0} \leq N\left\|\nabla e^{n-1}\right\|_{0}^{2}+C^{2} \lambda\left\|\nabla \eta^{n}\right\|_{0} \\
& \leq\left(N\left\|\nabla u_{h}^{n-1}\right\|_{0}+N\left\|\nabla u_{h}\right\|_{0}+C^{2} \lambda^{2} \bar{N} \varepsilon\right)\left\|\nabla e^{n-1}\right\|_{0} \\
& \leq\left(\frac{16 N C^{2} \lambda \varepsilon}{3 v}+C^{2} \lambda^{2} \bar{N} \varepsilon\right)\left\|\nabla e^{n-1}\right\|_{0}=\frac{7 v}{16}\left\|\nabla e^{n-1}\right\|_{0^{\prime}} \\
&\left\|\nabla e^{n}\right\|_{0} \leq \frac{1}{2}\left\|\nabla e^{n-1}\right\|_{0}
\end{aligned}
$$

From the inductive method, we know, for $n=1$, subtracting (2.10) from (2.9), we can get

$$
a\left(e^{1}, v_{h}\right)-d\left(\mu^{1}, v_{h}\right)+d\left(q_{h}, e^{1}\right)+b\left(u_{h}, u_{h}, v_{h}\right)+G\left(\mu^{1}, q_{h}\right)=\lambda\left(j T^{n}, v_{h}\right)
$$

Letting $v_{h}=e^{1}, q_{h}=\mu^{1}$ in (4.12) and using (2.14), we have

$$
\begin{aligned}
\left\|\nabla e^{1}\right\|_{0}+G\left(\mu^{1}, \mu^{1}\right) & \leq v^{-1} N\left\|\nabla u_{h}\right\|_{0}^{2}+v^{-1} C^{2} \lambda\left\|\nabla T_{h}\right\|_{0} \\
& \leq \frac{64 C^{4} \lambda^{2} N \varepsilon^{2}}{9 v^{3}}+\frac{C^{2} \lambda \varepsilon}{v} \leq \frac{4 C^{2} \lambda \varepsilon}{3 v},
\end{aligned}
$$

then

$$
\left\|\nabla e^{1}\right\|_{0} \leq \frac{4 C^{2} \lambda \varepsilon}{3 v}
$$

By (4.7), we have

$$
\left\|\nabla \eta^{1}\right\|_{0} \leq \lambda \bar{N} \varepsilon\left\|\nabla e^{n}\right\|_{0} \leq \lambda \bar{N} \frac{4 C^{2} \lambda \varepsilon^{2}}{3 v} \leq \frac{\varepsilon}{4}
$$

Letting $q_{h}=0$ in (4.12), (2.14), and (3.9), using Lemma 2.1, we get

$$
\beta\left\|\mu^{1}\right\|_{0} \leq v\left\|\nabla e^{1}\right\|_{0}+N\left\|\nabla u_{h}\right\|_{0}^{2}+C \lambda\left\|T_{h}\right\|_{0} \leq \frac{v \varepsilon}{2}+\frac{4 C^{2} \lambda \varepsilon}{3 v} .
$$

Assuming that (4.3) is true for $n=k-1$, using (4.7) and (4.11), we know that both of them are valid for $n=k$. Using (4.7) holds for $n=k$, we let $q_{h}=0$ in (4.4) and using Lemma 2.1, (4.5), and (3.3), we have

$$
\begin{aligned}
\beta\left\|\mu^{n}\right\|_{0} & \leq(v+2 N \varepsilon)\left\|\nabla e^{n}\right\|_{0}+N\left\|\nabla e^{n-1}\right\|_{0}^{2}+C^{2} \lambda\left\|\nabla \eta^{n-1}\right\|_{0} \\
& \leq(v+2 N \varepsilon) \frac{C^{2} \lambda \varepsilon}{2^{n-3} 3 v}+N\left(\frac{C^{2} \lambda \varepsilon}{2^{n-4} 3 v}\right)^{2}+\frac{C^{2} \lambda \varepsilon}{2^{n}} .
\end{aligned}
$$


Theorem 4.4. Under the assumptions of Theorem 4.3, then there holds that

$$
\begin{gathered}
\lim _{n \rightarrow \infty}\left(\left\|u_{h}^{n}-u_{h}^{n-1}\right\|_{0}+\left\|\nabla\left(u_{h}^{n}-u_{h}^{n-1}\right)\right\|_{0}\right)=0, \\
\left\|\nabla e^{n}\right\|_{0}+\left\|\mu^{n}\right\|_{0}+\left\|\nabla \eta^{n}\right\|_{0} \leq F|\log h|^{1 / 2}\left\|\nabla\left(u_{h}^{n}-u_{h}^{n-1}\right)\right\|_{0}\left\|u_{h}^{n}-u_{h}^{n-1}\right\|_{0}+\frac{H \varepsilon}{2^{n+1}},
\end{gathered}
$$

where $F$ and $H$ are two positive constants.

Proof. By using $\left(\mathrm{B}_{1}\right)$ and triangle inequality, we have

$$
\left\|u_{h}^{n}-u_{h}^{n-1}\right\|_{0}+\left\|\nabla\left(u_{h}^{n}-u_{h}^{n-1}\right)\right\|_{0} \leq(C+1)\left(\left\|\nabla e^{n}\right\|_{0}+\left\|\nabla e^{n-1}\right\|_{0}\right) .
$$

Using Theorem 4.3, letting $n \rightarrow \infty$, we obtain (4.18). Taking $v_{h}=e^{n}, q_{h}=\mu^{n}$ in (4.4) and using (2.14), we get

$$
a\left(e^{n}, e^{n}\right)+b\left(e^{n}, u_{h}^{n-1}, e^{n}\right)+G\left(\mu^{n}, \mu^{n}\right)=-b\left(u_{h}^{n}-u_{h}^{n-1}, u_{h}^{n}-u_{h}^{n-1}, e^{n}\right)+\lambda\left(j \eta^{n}, e^{n}\right) .
$$

By (2.14) and Lemma 3.1, we deduce

$$
\left(v-N\left\|u_{h}^{n-1}\right\|_{0}\right)\left\|\nabla e^{n}\right\|_{0}+G\left(\mu^{n}, \mu^{n}\right) \leq F|\log h|^{1 / 2}\left\|\nabla\left(u_{h}^{n}-u_{h}^{n-1}\right)\right\|\left\|_{0}\right\| u_{h}^{n}-u_{h}^{n-1}\left\|_{0}+F^{2} \lambda\right\| \nabla \eta^{n} \|_{0} .
$$

Combining (3.3) and (4.7), we obtain

$$
\left(v-\frac{8 N \varepsilon}{3 \mathcal{v}}\right)\left\|\nabla e^{n}\right\|_{0} \leq F|\log h|^{1 / 2}\left\|\nabla\left(u_{h}^{n}-u_{h}^{n-1}\right)\right\|_{0}\left\|u_{h}^{n}-u_{h}^{n-1}\right\|_{0}+F^{2} \lambda^{2} \bar{N} \varepsilon\left\|\nabla e^{n-1}\right\|_{0} .
$$

Using (3.2), we get

$$
\left\|\nabla e^{n}\right\|_{0} \leq F|\log h|^{1 / 2}\left\|\nabla\left(u_{h}^{n}-u_{h}^{n-1}\right)\right\|_{0}\left\|u_{h}^{n}-u_{h}^{n-1}\right\|_{0}+\frac{H \varepsilon}{2^{n+1}}
$$

Combining (3.2), (4.7), and (4.17), we get

$$
\begin{aligned}
& \left\|\nabla \eta^{n}\right\|_{0} \leq F|\log h|^{1 / 2}\left\|\nabla\left(u_{h}^{n}-u_{h}^{n-1}\right)\right\|_{0}\left\|u_{h}^{n}-u_{h}^{n-1}\right\|_{0}+\frac{H \varepsilon}{2^{n+1}}, \\
& \left\|\nabla \mu^{n}\right\|_{0} \leq F|\log h|^{1 / 2}\left\|\nabla\left(u_{h}^{n}-u_{h}^{n-1}\right)\right\|_{0}\left\|u_{h}^{n}-u_{h}^{n-1}\right\|_{0}+\frac{H \varepsilon}{2^{n+1}} .
\end{aligned}
$$

Here, we complete the proof. 
Theorem 4.5. Under the assumptions of Theorem 4.3, the following inequality:

$$
\begin{aligned}
& \left\|\nabla\left(u-u_{h}^{n}\right)\right\|_{0}+\left\|p-p_{h}^{n}\right\|_{0}+\left\|\nabla\left(T-T_{h}^{n}\right)\right\|_{0} \leq F_{1} h^{m}\left(\|u\|_{m+1}+\|p\|_{m}+\|T\|_{m+1}\right) \\
& \quad+F|\log h|^{1 / 2}\left\|\nabla\left(u_{h}^{n}-u_{h}^{n-1}\right)\right\|_{0}\left\|u_{h}^{n}-u_{h}^{n-1}\right\|_{0}+\frac{H \varepsilon}{2^{n+1}}
\end{aligned}
$$

holds, where $F_{1}$ and $H$ are the positive constants.

Proof. By Lemma 4.1, Theorem 4.4, and the triangle inequality, this theorem is obviously true.

\section{Numerical Test}

This section presents the numerical results that complement the theoretical analysis.

\subsection{Convergence Analysis}

In our experiment, $\Omega=[0,1] \times[0,1]$ is the unit square in $R^{2}$. Let $T_{0}=0$ on left and lower boundary of the cavity, $\partial T / \partial n=0$ on upper boundary of the cavity, and $T_{0}=4 y(1-y)$ on right boundary of the cavity (see Figure 1 ). Physics model of the cavity flows: $t=0$, that is, $n=0$ initial values on boundary. In general, we cannot know the exact solution of the stationary conduction-convection equations. In order to get the exact solution, we design the procedure as follows. Firstly, solving the stationary conduction-convection equations by using the $P_{2}-P_{1}-P_{2}$ finite element pair, which holds stability, on the finer mesh, we take the solution as the exact solution. Secondly, the absolute error is obtained by comparing the exact solution and the finite element solutions with VMS methods. Finally, we can easily obtain errors and convergence rates.

\subsection{Driven Cavity}

In this experiment, $\Omega=[0,1] \times[0,1]$ is the unit square in $R^{2}$. Let $T_{0}=0$ on left and lower boundary of the cavity, $\partial T / \partial n=0$ on upper boundary of the cavity, and $T_{0}=4 y(1-y)$ on right boundary of the cavity (see Figure 1 ). Physics model of the cavity flows: $t=0$, that is, $n=0$ initial values on boundary. Solving the stationary conduction-convection equations by using the $P_{2}-P_{1}-P_{2}$ finite element pair, which holds stability results, on the finer mesh, we take the solution as the exact solution. From Figures 1 and 2, we know that the solution of finite element VMS using $P_{1}-P_{1}-P_{1}$ element agree completely with the "exact solution." In Figure 3, we choose $\operatorname{Re}=2000$, divide the cavity into $M \times N=100 \times 100$, from left to right shows the numerical streamline, the numerical isobar, and the numerical isotherms. In Figure 4, we choose $\operatorname{Re}=3000$, divide the cavity into $M \times N=100 \times 100$, from left to right shows the numerical streamline, the numerical isobar, and the numerical isotherms.

Remark 5.1. Our VMS finite element method based on two local Gauss integrations and $\varepsilon_{d}=$ $0.1 \mathrm{~h}$ is suitable for the Sobolev space. Throughout the paper, our analysis and numerical tests are all carried out for the $P_{1}-P_{1}-P_{1}$ element (see Tables 1 and 2). 

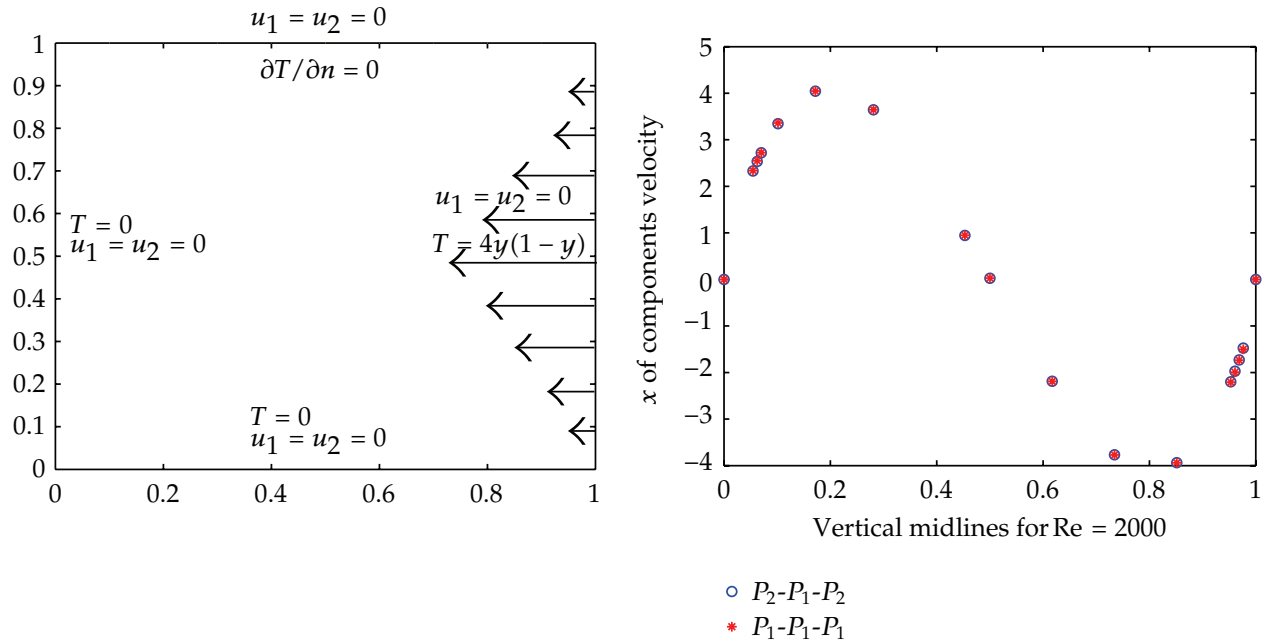

(a)

(b)

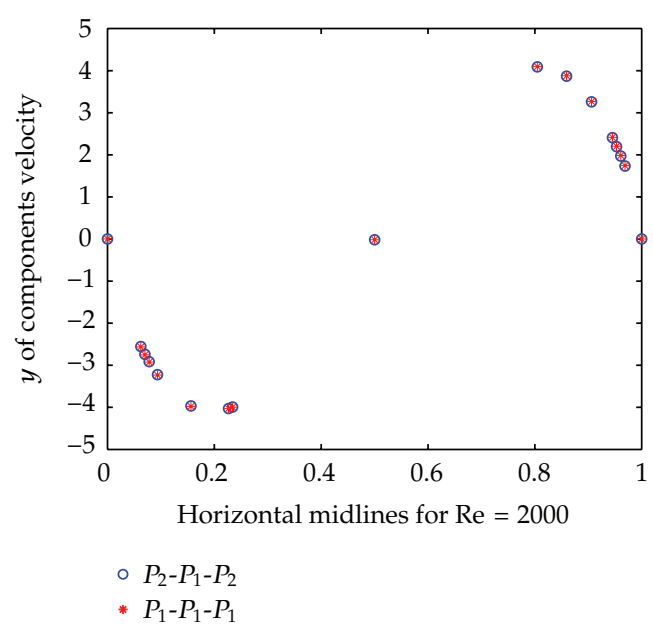

(c)

Figure 1: From (a) to (c): physics model of the cavity flows, vertical midlines for $\operatorname{Re}=2000, h=1 / 100$, horizontal midlines for $\operatorname{Re}=2000, h=1 / 100$.

\section{Conclusion}

In this paper, we studied a finite element VMS algorithm based on two local Gauss integrations to solve the stationary conduction-convection problem. From Figures 1 and 2, we see that the solution of VMS using $P_{1}-P_{1}-P_{1}$ and $\varepsilon_{d}=0.1 \mathrm{~h}$ agrees completely with the "exact solution," which shows that our method is highly efficient for the stationary conductionconvection problems. Numerical tests tell us that VMS finite element method based on two local Gauss integrations is very effective. 


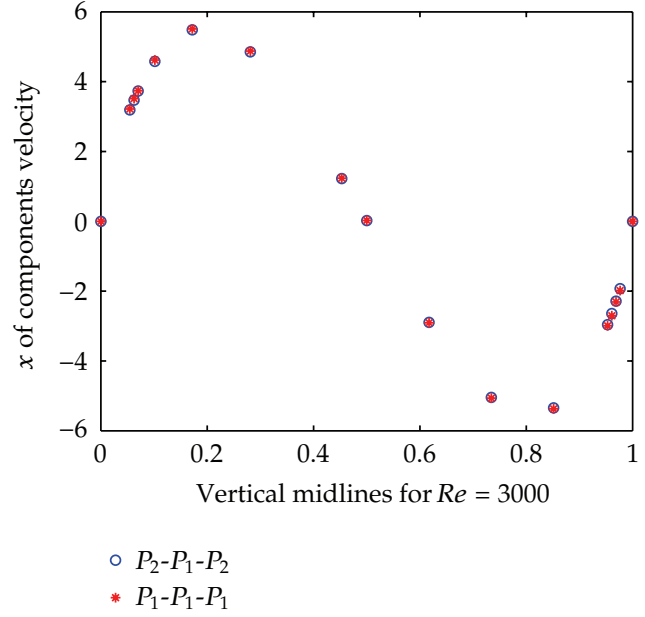

(a)

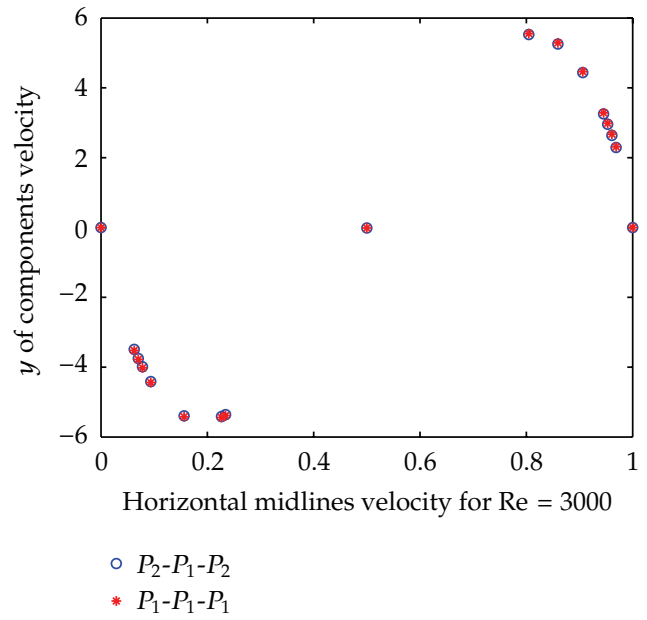

(b)

Figure 2: From (a) to (b): vertical midlines for $\operatorname{Re}=3000, h=1 / 100$, horizontal midlines for $\operatorname{Re}=3000, h=$ $1 / 100$.

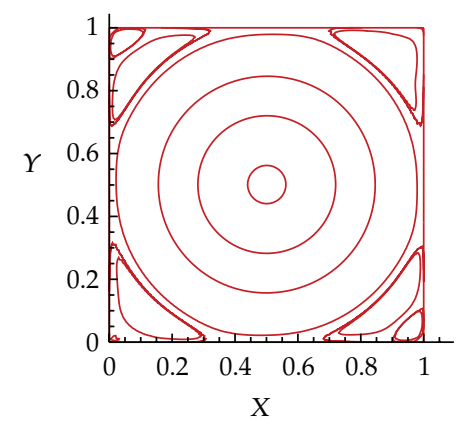

(a)

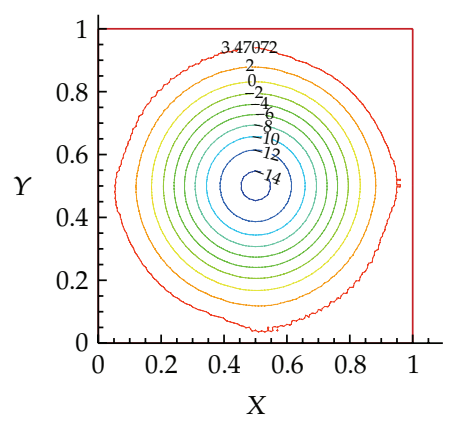

(b)

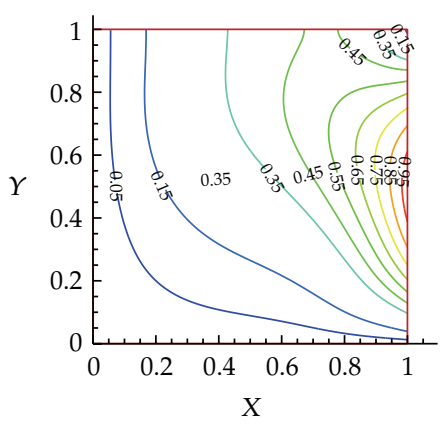

(c)

Figure 3: For $\operatorname{Re}=2000, h=1 / 100$, from (a) to (c): velocity streamlines, the pressure level lines, numerical isotherms.

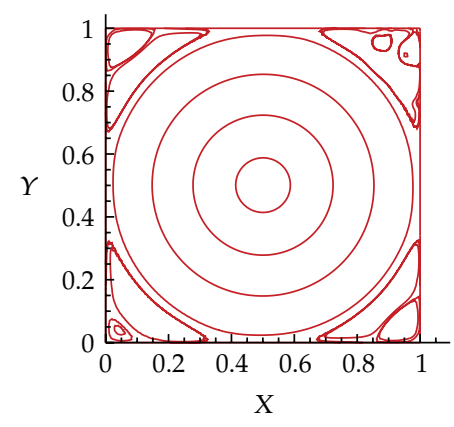

(a)

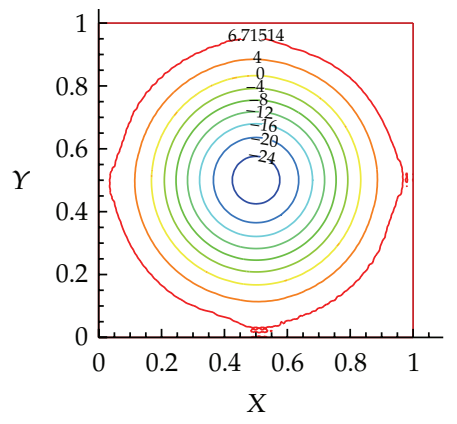

(b)

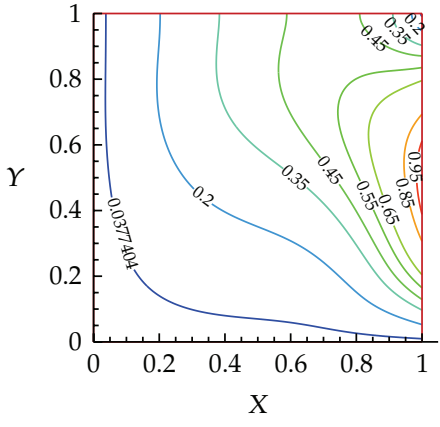

(c)

Figure 4: For $\operatorname{Re}=3000, h=1 / 100$, from (a) to (c): velocity streamlines, the pressure level lines, numerical isotherms. 
Table 1: VMS: $P_{1}-P_{1}-P_{1}$ element.

\begin{tabular}{lccccc}
\hline $1 / h$ & $\left\|u-u_{h}\right\|_{0}$ & $\left\|u-u_{h}\right\|_{1}$ & $\left\|T-T_{h}\right\|_{0}$ & $\left\|T-T_{h}\right\|_{1}$ & $\left\|p-p_{h}\right\|_{0}$ \\
\hline 10 & 0.000194122 & 0.00493006 & 0.00740049 & 0.277241 & 0.00506075 \\
20 & $4.91998 e-005$ & 0.00252269 & 0.00208824 & 0.153561 & 0.00308312 \\
40 & $1.21288 e-005$ & 0.00126459 & 0.0005746 & 0.0838877 & 0.00180539 \\
60 & $5.35135 e-006$ & 0.000842093 & 0.000266991 & 0.0583954 & 0.00131444 \\
80 & $2.98429 e-006$ & 0.000630808 & 0.000154418 & 0.0457979 & 0.00105007 \\
\hline
\end{tabular}

Table 2: VMS: $P_{1}-P_{1}-P_{1}$ element.

\begin{tabular}{lccccc}
\hline $1 / h$ & $u_{L^{2}}$ rate & $u_{H^{1}}$ rate & $T_{L^{2}}$ rate & $T_{H^{1}}$ rate & $p_{L^{2}}$ rate \\
\hline 10 & $/$ & $/$ & $/$ & $/$ & $/$ \\
20 & 1.9802 & 0.9666 & 1.8253 & 0.8523 & 0.7150 \\
40 & 2.0202 & 0.9963 & 1.8617 & 0.8723 & 0.7721 \\
60 & 2.0180 & 1.0028 & 1.8903 & 0.8934 & 0.7827 \\
80 & 2.0300 & 1.0042 & 1.9033 & 0.8447 & 0.7806 \\
\hline
\end{tabular}

\section{Acknowledgments}

The project is supported by NSF of China (10971164) and the Research Foundation of Xianyang Normal University (06xsyk265).

\section{References}

[1] K. Chida, "Surface temperature of a flat plate of finite thickness under conjugate laminar forced convection heat transfer condition," International Journal of Heat and Mass Transfer, vol. 43, no. 4, pp. 639-642, 1999.

[2] J. A. M. García, J. M. G. Cabeza, and A. C. Rodríguez, "Two-dimensional non-linear inverse heat conduction problem based on the singular value decomposition," International Journal of Thermal Sciences, vol. 48, no. 6, pp. 1081-1093, 2009.

[3] D. C. Kim and Y. D. Choi, "Analysis of conduction-natural convection conjugate heat transfer in the gap between concentric cylinders under solar irradiation," International Journal of Thermal Sciences, vol. 48 , no. 6, pp. 1247-1258, 2009.

[4] Z. D. Luo, The Bases and Applications of Mixed Finite Element Methods, Science Press, Beijing, China, 2006.

[5] Z. D. Luo and X. M. Lu, "A least-squares Galerkin/Petrov mixed finite element method for stationary conduction-convection problems," Mathematica Numerica Sinica, vol. 25, no. 2, pp. 231-244, 2003.

[6] C. P. Naveira, M. Lachi, R. M. Cotta, and J. Padet, "Hybrid formulation and solution for transient conjugated conduction-external convection," International Journal of Heat and Mass Transfer, vol. 52, no. 1-2, pp. 112-123, 2009.

[7] Q. W. Wang, M. Yang, and W. Q. Tao, "Natural convection in a square enclosure with an internal isolated vertical plate," Wärme-und Stoffübertragung, vol. 29, no. 3, pp. 161-169, 1994.

[8] M. Yang, W. Q. Tao, Q. W. Wang, and S. S. Lue, “On identical problems of natural convection in enclosures and applications of the identity character," Journal of Thermal Science, vol. 2, no. 2, pp. 116-125, 1993.

[9] E. DiBenedetto and A. Friedman, "Conduction-convection problems with change of phase," Journal of Differential Equations, vol. 62, no. 2, pp. 129-185, 1986.

[10] M. A. Christon, P. M. Gresho, and S. B. Sutton, "Computational predictability of time-dependent natural convection flows in enclosures (including a benchmark solution)," International Journal for Numerical Methods in Fluids, vol. 40, no. 8, pp. 953-980, 2002.

[11] M. S. Mesquita and M. J. S. de Lemos, "Optimal multigrid solutions of two-dimensional convectionconduction problems," Applied Mathematics and Computation, vol. 152, no. 3, pp. 725-742, 2004. 
[12] N. B. Cheikh, B. B. Beya, and T. Lili, "A multigrid method for solving the Navier-Stokes/Boussinesq equations," Communications in Numerical Methods in Engineering, vol. 24, no. 8, pp. 671-681, 2008.

[13] Z. Luo, J. Chen, I. M. Navon, and J. Zhu, "An optimizing reduced PLSMFE formulation for nonstationary conduction-convection problems," International Journal for Numerical Methods in Fluids, vol. 60, no. 4, pp. 409-436, 2009.

[14] Z. Si, T. Zhang, and K. Wang, "A Newton iterative mixed finite element method for stationary conduction-convection problems," International Journal of Computational Fluid Dynamics, vol. 24, no. 3, pp. 135-141, 2010.

[15] Z. Si and Y. He, "A defect-correction mixed finite element method for stationary conduction-convection problems," Mathematical Problems in Engineering, vol. 2011, Article ID 370192, 28 pages, 2011.

[16] J. Boland and W. Layton, "Error analysis for finite element methods for steady natural convection problems," Numerical Functional Analysis and Optimization, vol. 11, no. 5-6, pp. 449-483, 1990.

[17] J. L. Guermond, "Stabilization of Galerkin approximations of transport equations by subgrid modelling," Mathematical Modelling and Numerical Analysis, vol. 33, no. 6, pp. 1293-1316, 1999.

[18] T. J. R. Hughes, L. Mazzei, A. A. Oberai, and A. A. Wray, "The multiscale formulation of large eddy simulation: decay of homogeneous isotropic turbulence," Physics of Fluids, vol. 13, no. 2, pp. 505-512, 2001.

[19] T. Hughes, L. Mazzei, and K. Jansen, "Large eddy simulation and the variational multiscale method," Computing and Visualization in Science, vol. 3, no. 1-2, pp. 47-59, 2000.

[20] T. J. R. Hughes, “Multiscale phenomena: green's functions, the Dirichlet-to-Neumann formulation, subgrid scale models, bubbles and the origins of stabilized methods," Computer Methods in Applied Mechanics and Engineering, vol. 127, no. 1-4, pp. 387-401, 1995.

[21] W. Layton, "A connection between subgrid scale eddy viscosity and mixed methods," Applied Mathematics and Computation, vol. 133, no. 1, pp. 147-157, 2002.

[22] V. John and S. Kaya, "A finite element variational multiscale method for the Navier-Stokes equations," SIAM Journal on Scientific Computing, vol. 26, no. 5, pp. 1485-1503, 2005.

[23] H. Zheng, Y. Hou, F. Shi, and L. Song, "A finite element variational multiscale method for incompressible flows based on two local gauss integrations," Journal of Computational Physics, vol. 228, no. 16, pp. 5961-5977, 2009.

[24] A. Masud and R. A. Khurram, "A multiscale finite element method for the incompressible NavierStokes equations," Computer Methods in Applied Mechanics and Engineering, vol. 195, no. 13-16, pp. 1750-1777, 2006.

[25] J. Li, Y. He, and Z. Chen, "A new stabilized finite element method for the transient Navier-Stokes equations," Computer Methods in Applied Mechanics and Engineering, vol. 197, no. 1-4, pp. 22-35, 2007.

[26] J. Li and Y. He, "A stabilized finite element method based on two local Gauss integrations for the Stokes equations," Journal of Computational and Applied Mathematics, vol. 214, no. 1, pp. 58-65, 2008. 


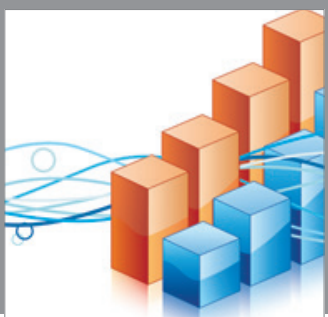

Advances in

Operations Research

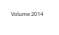

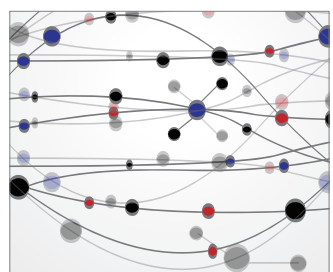

\section{The Scientific} World Journal
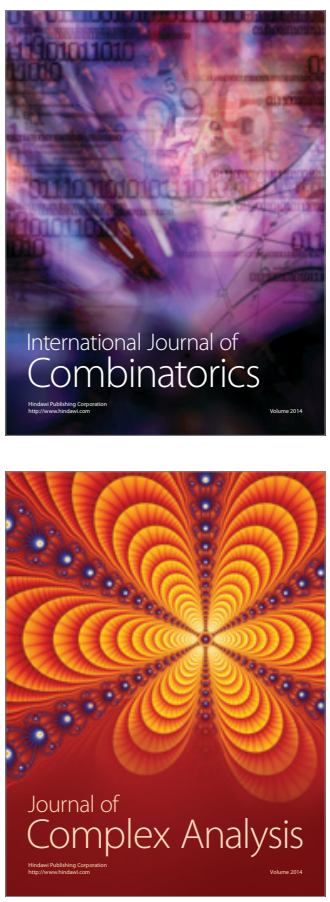

International Journal of

Mathematics and

Mathematical

Sciences
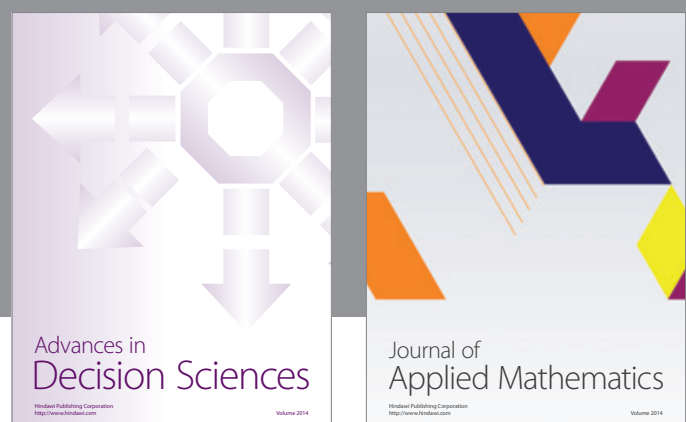

Journal of

Applied Mathematics
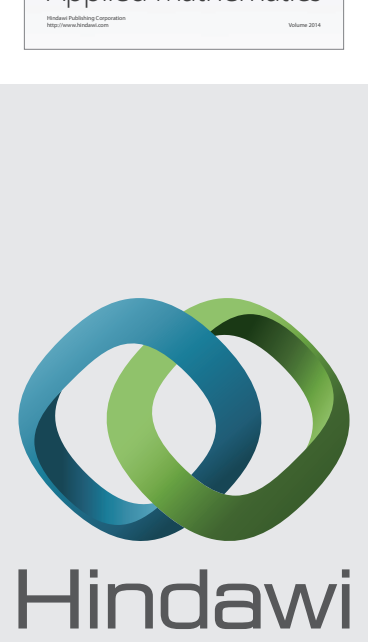

Submit your manuscripts at http://www.hindawi.com
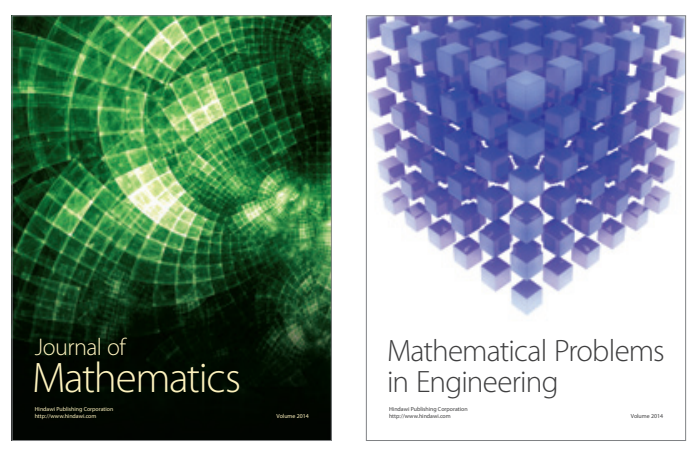

Mathematical Problems in Engineering
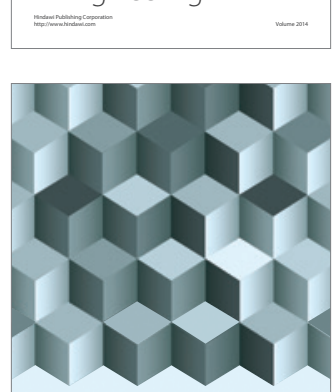

Journal of

Function Spaces
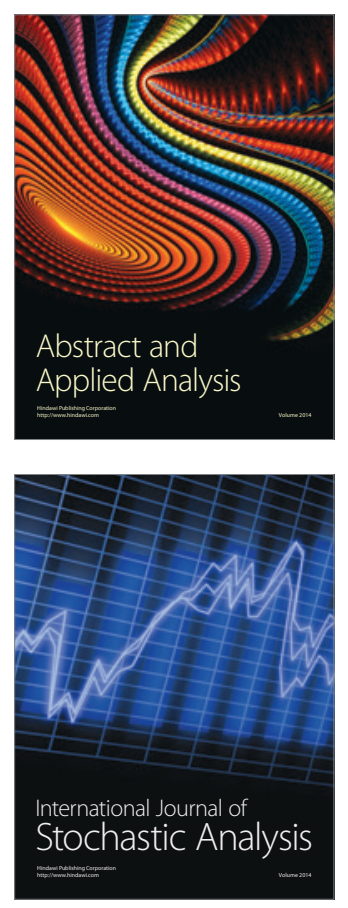

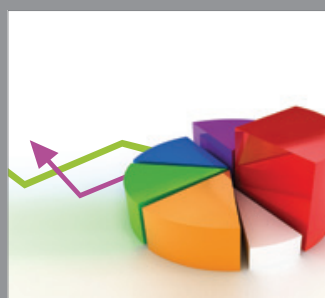

ournal of

Probability and Statistics

Promensencen
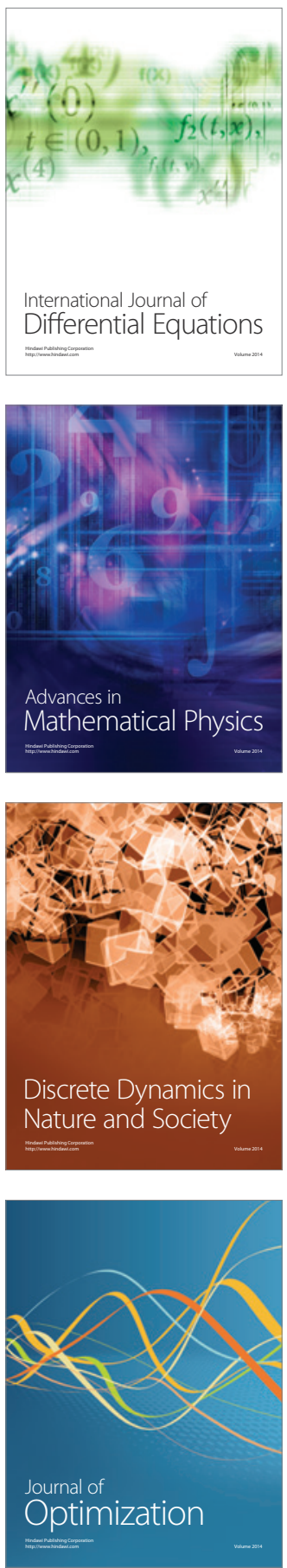